Published in final edited form as:

Fertil Steril. 2018 January ; 109(1): 137-141.e2. doi:10.1016/j.fertnstert.2017.09.029.

\title{
Kisspeptin as a New Serum Biomarker to Discriminate Miscarriage from Viable Intrauterine Pregnancy
}

\author{
Chantae Sullivan-Pyke, M.D. ${ }^{a}$, Daniel J Haisenleder, Ph.D. ${ }^{b}$, Suneeta Senapati, M.D., \\ MSCE $^{a}$, Olivia Nicolais, B.A. ${ }^{a}$, Esther Eisenberg, M.D., MPH ${ }^{d}$, Mary D. Sammel, ScD ${ }^{a, c}$, and \\ Kurt T. Barnhart, M.D., MSCE ${ }^{a, c}$ \\ aDepartment of Obstetrics and Gynecology, University of Pennsylvania \\ bUniversity of Virginia Center for Research in Reproduction \\ 'Department of Biostatistics and Epidemiology Perelman School of Medicine, University of \\ Pennsylvania, Philadelphia, PA \\ ${ }^{\mathrm{d} R e p r o d u c t i v e ~ M e d i c i n e ~ N e t w o r k, ~ F e r t i l i t y ~ a n d ~ I n f e r t i l i t y ~ B r a n c h, ~ E u n i c e ~ K e n n e d y ~ S h r i v e r ~ N a t i o n a l ~}$ \\ Institute of Child Health and Human Development, $\mathrm{NIH}$
}

\begin{abstract}
Objective-To validate the ability of serum kisspeptin-54 to discriminate between first trimester viable pregnancies and miscarriages.

Design-Case-control study.

Setting-Academic medical centers.

Patients-Women with confirmed viable intrauterine pregnancy (IUP) with estimated gestational age 6 - 10 weeks $(n=20)$, women with confirmed miscarriage (SAB) between estimated gestational age $6-10$ weeks $(n=20)$, and non-pregnant women $(n=19)$.

Intervention(s)—Collection of serum samples from women with confirmed IUP, SAB, and nonpregnant women for the measurement of serum kisspeptin and serum human chorionic gonadotropin (hCG) hormone levels.

Main Outcome Measure(s)-Serum kisspeptin and hCG.

Results-The limit of detection was $0.024 \mathrm{ng} / \mathrm{ml}$; intra- and inter-assay coefficients of variation were $5.1 \%$ and $8.6 \%$, respectively. Kisspeptin levels differed between the pregnant and nonpregnant state (median $1.50 \mathrm{ng} / \mathrm{mL}$, range $0.55-3.72$, $\mathrm{p}<0.0001$ ) and by viability (median 0.20 $\mathrm{ng} / \mathrm{mL}$, range $0.07-0.37, \mathrm{p}<0.0001$ ). Kisspeptin levels are positively associated with gestational age ( $\mathrm{p}<0.001)$. There was also a significant positive association with $\mathrm{hCG}$ in SAB $(\mathrm{p}=0.032)$, but not IUP $(\mathrm{p}=0.778)$.
\end{abstract}

Corresponding Author: Kurt Barnhart, MD, MSCE., Address: 3701 Market Street, Suite 800, Philadelphia, PA 19104, KBarnhart@uphs.upenn.edu, Telephone number: 215-662-2974, Fax number: 215-349-5512.

Publisher's Disclaimer: This is a PDF file of an unedited manuscript that has been accepted for publication. As a service to our customers we are providing this early version of the manuscript. The manuscript will undergo copyediting, typesetting, and review of the resulting proof before it is published in its final citable form. Please note that during the production process errors may be discovered which could affect the content, and all legal disclaimers that apply to the journal pertain. 
Conclusion-Plasma levels of kisspeptin have been suggested as a biomarker for miscarriage. This study demonstrates kisspeptin assay stability in serum and its potential clinical utility as a biomarker for early pregnancy viability.

\section{Keywords}

kisspeptin; biomarker; intrauterine pregnancy; miscarriage

\section{Introduction}

Miscarriage or spontaneous abortion (SAB) affects 10-20\% of clinical pregnancies and this rate is highest prior to 12 weeks gestational age (1). SAB may occur due to embryonic aneuploidy, intrauterine infection, thrombotic event, abnormal placentation and, in many cases, the cause is unexplained (2).

Early pregnancy viability is currently assessed by trends of sequential serum hCG values and transvaginal ultrasound (TVUS) measurements. However, a normally rising hCG level does not necessarily predict pregnancy viability in women presenting with bleeding in the first trimester (3). An emerging biomarker for the discrimination of viable pregnancy is kisspeptin, due to its potential regulatory role in trophoblast function and placentation $(6,7)$. While the performance of kisspeptin as a plasma biomarker to discriminate SAB and IUP in the late first trimester has been demonstrated (7), its utility as a serum marker in the early first trimester is unknown, and would be important for clinical application.

The aim of the study was to validate a serum kisspeptin-54 assay and assess its ability to discriminate between early first trimester viable pregnancies and $\mathrm{SAB}$ in a population of women with symptomatic pregnancy.

\section{Materials and Methods}

This study is a nested case-control design including 40 women who presented with symptoms of bleeding and/or cramping to the Emergency Room at one of three academic medical centers prior to their initial prenatal visit. Patients presented between September 2013 and February 2016.

Women were considered for the study if they presented between 6-10 weeks gestational age by last menstrual period with abdominal pain, vaginal bleeding or both. Patients were included if they had either confirmed intrauterine pregnancy (IUP) by ultrasound $(n=20)$ or confirmed miscarriage (SAB) by ultrasound $(n=20)$. Patients were excluded from the study if they had a diagnosis of ectopic pregnancy, molar pregnancy, multiple gestations, gestational age past 12 weeks at the time of blood draw or if pregnancies were assisted conceptions. The serum and plasma samples were obtained from the Serum and Plasma Bank for the Development of Novel Ectopic Pregnancy Biomarkers, an ongoing multi-center prospective cohort study at the University of Pennsylvania (PENN), Eastern Virginia Medical School (EVMS), and Northwestern University (NW). Serum was also collected from 19 non-pregnant women as controls. Institutional Review Board approval was obtained at each site. 
SAB was defined as the presence of gestational sac $\geq 25 \mathrm{~mm}$ on transvaginal ultrasound (TVUS) without evidence of embryonic pole, embryonic pole $\geq 7 \mathrm{~mm}$ without cardiac activity (4), inappropriate growth of a gestational sac with no further development of the pregnancy, the absence of embryonic cardiac activity after previously documented embryonic cardiac activity, or downtrending hCG after presenting with vaginal bleeding. Pregnancies were followed to term when possible or until at least 12 weeks with the presence of cardiac activity to ensure IUPs were ongoing.

Kisspeptin-54 was measured in serum at the core laboratory of the Reproductive Medicine Network using a commercial ELISA (Peninsula Laboratories International, Inc.; San Carlos, CA). The assay was validated for non-extracted serum samples by spiking a commercial human serum pool (Sigma Chemical; St Louis, MO) with various concentrations of recombinant human Kisspeptin-54 (provided by the kit manufacturer), and demonstrating parallelism to the assay standard curve (5). Serum hCG concentrations were determined with the Abbot Axsym total beta immunoassay (Abbot Laboratories), and results are expressed as $\mathrm{mIU} / \mathrm{mL}$, using the Third International Reference Preparation (6).

\section{Statistics}

Pearson's chi-squared test was used to evaluate between group differences by pregnancy outcome for categorical data including race and ethnicity. Student's $t$ test was used to evaluate differences between group means by pregnancy outcome for continuous data such as gestational age. Linear regression was used to evaluate whether maternal age varied with pregnancy outcome. Differences in serum biomarker levels and maternal age by pregnancy outcome were assessed by Kruskal-Wallis or Wilcoxon rank-sum test as appropriate. Kisspeptin levels were correlated with serum human chorionic gonadotropin (hCG) levels, to assess applicability in the context of current standard of care. Data were log transformed for analysis, and linear regression was used to estimate the association between serum kisspeptin and hCG in IUPs and SABs. All statistics were carried out with STATA v14.2 (StataCorp LLC., College Station, TX). A p value $<0.05$ was considered statistically significant.

\section{Results}

The demographic characteristics of the study population are shown in Table 1. Women who experienced miscarriage presented at a significantly lower gestational age than women who had ongoing intrauterine pregnancies (mean IUP gestational age in days 68.1 \pm 9.9 , mean SAB gestational age in days $52.7 \pm 10.0, \mathrm{p}<0.001)$. The racial and ethnic distribution reflects the urban population of patients presenting to the emergency rooms of the academic centers during the study. The majority of the patients in the IUP and SAB groups were from the PENN accounting for $60 \%$ of these patients, $27.5 \%$ were from NW, and $12.5 \%$ of patients were from EVMS. Among the PENN patients, $87.5 \%$ were African American, compared to only $4.2 \%$ African American at NW and 8.3\% at EVMS. Mean maternal age is $30.9 \pm 6.4$ years. There is a statistically significant difference in maternal age between the groups (IUP $27.1 \pm 4.9$ years, $\mathrm{SAB} 30.4 \pm 7.1$ years, non-pregnant $35.5 \pm 3.9$ years, $\mathrm{p}<0.001$ ), but the women in the IUP and SAB groups fall in the $20-30$ year-old age group in which the risk 
of miscarriage is known to be $9-17 \%$, making them similar from a clinical perspective (7). The women who served as non-pregnant controls were older than the other two groups (Table 1).

Kisspeptin assay characteristics and discriminatory performance are shown in Table 2 and Figure 1. The limit of detection was $0.024 \mathrm{ng} / \mathrm{ml}$; intra-assay and inter-assay coefficients of variation were $5.1 \%$ and $8.6 \%$, respectively. Kisspeptin is detectable in serum in the nonpregnant state, and the levels are significantly lower in comparison to pregnant women ( $\mathrm{p}<$ 0.0001). Table 2 compares median serum kisspeptin and hCG levels in IUPs versus SABs and in the pregnant versus nonpregnant state. Serum kisspeptin levels are higher in IUP (median $1.50 \mathrm{ng} / \mathrm{mL}$, range $0.55-3.72, \mathrm{p}<0.0001$ ) compared to SAB (median $0.20 \mathrm{ng} / \mathrm{mL}$, range $0.07-0.37, \mathrm{p}<0.0001$ ). Similarly, hCG levels are higher in confirmed IUP (median $117202 \mathrm{mIU} / \mathrm{mL}$, range 83975 - 148784) compared to SAB (median $4739 \mathrm{mIU} / \mathrm{mL}$, range 1858 - 8650) (Table 2). Linear regression between logkisspeptin and covariates demonstrate that serum kisspeptin levels are positively associated with gestational age $(\mathrm{p}<0.001)$. When gestational age is controlled for, kisspeptin levels are still significantly higher in IUPs than SABs ( $p<0.001)$. No significant association was seen between serum kisspeptin levels and race $(p=0.117)$, ethnicity $(p=0.542)$, or maternal age $(p=0.255)$ among IUPs and SABs. Receiver operator characteristic (ROC) curve analysis demonstrates excellent accuracy of the kisspeptin assay in discriminating SAB from IUP at the time of diagnosis (AUC 0.95 [95\% CI 0.89 - 1.00], Figure 1.

To determine whether there was a relationship between kisspeptin and hCG depending on pregnancy state, a linear regression analysis was performed (Supplemental Figure 1). The linear regression analysis indicates that hCG and kisspeptin are positively associated in SABs ( $\mathrm{p}=0.032$ ), but there is no significant association amongst the levels of kisspeptin and hCG in IUP $(\mathrm{p}=0.778)$. Kisspeptin levels are also higher in IUPs compared to SABs ( $<$ < $0.001)$.

Serum and plasma kisspeptin values were also compared in a subgroup of the study population. Plasma was available for 8 IUP patients and 3 SAB patients. Median plasma kisspeptin in IUP was $3.52 \mathrm{ng} / \mathrm{mL}$ (range 1.09 to $21.7 \mathrm{ng} / \mathrm{mL}$ ) while the median plasma kisspeptin in SAB was $2.7 \mathrm{ng} / \mathrm{mL}$ (range 0.46 to $2.7 \mathrm{ng} / \mathrm{mL}$ ), revealing a trend toward higher plasma kisspeptin values in IUP compared to SAB (Figure 2).

\section{Discussion}

Miscarriage is a common problem with a potentially devastating impact on patients.

However, our current tools for evaluating patients with concerning symptoms in the first trimester of pregnancy do not sufficiently discriminate between normal or abnormal pregnancy outcome in these patients. Kisspeptin, which is a known regulator of puberty, and which is found in high levels in the placenta, is an emerging biomarker in the evaluation of placental dysfunction and miscarriage $(8,9)$.

Kisspeptin is a family of peptide hormones encoded by the KISS1 gene. Kisspeptin acts centrally via the kisspeptin receptor to stimulate the release of gonadotropin releasing 
hormone $(\mathrm{GnRH})$ from the hypothalamus and has a critical role in the initiation of puberty $(8,10)$. The most abundant kisspeptin in human circulation is kisspeptin-54 and it is this form of kisspeptin that has been investigated as a biomarker for pregnancy viability (11). Kisspeptin was first discovered as an inhibitor of tumor metastasis and, as a result, was previously known as metastatin (12). Kisspeptin is also found in high levels in first trimester syncytiotrophoblast cells (13). Syncytiotrophoblast cells secrete matrix metalloproteinases (MMP) that degrade extracellular matrix and make invasion possible, however, kisspeptin has been shown in in vitro studies, to block the migration of trophoblasts by suppressing the action of MMPs (14-16). Kisspeptin levels rise with gestational age during pregnancy, but relatively low circulating levels of kisspeptin are seen in pregnancies complicated by preeclampsia compared to normal pregnancies, indicating a role for kisspeptin as a marker of placental dysfunction $(13,17,18)$.

Jayasena et al. evaluated kisspeptin-54 as a biomarker to discriminate between viable pregnancy and miscarriage using an in-house radioimmunoassay developed in their laboratory and measured plasma kisspeptin drawn at the initial prenatal visit (11). They studied 993 asymptomatic women at the time of initial prenatal visit with mean GA 11.2 \pm 2.0 weeks. They found that single measurement of serum $\mathrm{hCG}$ or plasma kisspeptin at the initial prenatal visit were both able discriminate between viable and non-viable pregnancies, but plasma kisspeptin was more predictive. Our study evaluated the utility of serum kisspeptin in women presenting with symptomatic pregnancy in the first trimester prior to the initial prenatal visit to facilitate earlier detection of nonviable pregnancy.

Both plasma and serum assays are used clinically, but serum has been shown to be more sensitive for biomarker detection (19). We have shown that serum kisspeptin is present in non-pregnant patients at low levels, but that the levels are significantly higher in viable pregnancy. Furthermore, the serum kisspeptin assay was shown to be stable, unlike prior studies which showed rapid degradation in serum (20). The differences in kisspeptin levels between IUP versus SAB may be related to gestational age as the average gestational age among IUPs was higher than that in SABs (Table 1). Receiver operator characteristic (ROC) curve analysis demonstrated that the serum kisspeptin assay can discriminate SAB from IUP. The level of kisspeptin continues to rise throughout gestation into the third trimester and, unlike HCG, does not plateau. Perhaps a relationship was not seen because, in this study, we looked at only women in the first trimester and not beyond when the HCG curve would plateau and kisspeptin would continue to increase. Linear regression also showed that there is no significant correlation amongst the levels of kisspeptin and hCG in viable IUP, while there is a significant correlation between kisspeptin and hCG levels in SABs. Together, these results suggest that hCG and kisspeptin may be markers of different biological pathways related to the establishment of early pregnancy. Kisspeptin may therefore offer independent information that may potentially improve diagnostic accuracy beyond the use of $\mathrm{hCG}$ and TVUS alone in the clinical setting.

Sixteen of the 20 patients with confirmed IUPs in the study went on to deliver at term, one patient had a miscarriage at a gestational age of 14 weeks and 3 days, and 3 patients were lost to follow-up prior to the ascertainment of pregnancy outcome. The patient who had a second trimester miscarriage had a kisspeptin value of $8.07 \mathrm{ng} / \mathrm{mL}$ at 9 weeks gestation, 
which was higher than the median kisspeptin value in the IUP group. Given that only one patient in the IUP group had a second trimester miscarriage, we cannot draw conclusions and there may be other clinical factors contributing to a second trimester miscarriage that cannot be predicted by kisspeptin level. The range of kisspeptin values in patients who went on to have a normal pregnancy and delivery compared to those whose pregnancies ended in miscarriage is unaffected when the analysis is repeated excluding the IUP patient who had a second trimester miscarriage and those lost to follow-up (Supplementary Figure 2).

The strengths of the study lie in the fact that serum was collected earlier in this study than in prior studies, thus confirming the assay performance is valid in patients presenting early in gestation with symptomatic pregnancies. Additionally, the study of the kisspeptin assay in a well-phenotyped prospective cohort allows for outcome verification, though there is an inherent selection bias as a result. Limitations of the study include the small sample size and the cross-sectional nature of the study. Though the sample size is small, the case-control design with well-phenotyped patients in each group is appropriate for biomarker characterization and allowing for differences in serum kisspeptin between the groups to be detected if indeed differences existed (21). The findings from this study set the stage for further biomarker validation in larger prospective studies. Only patients with unassisted pregnancies were included, therefore applicability to patients with assisted conceptions is unknown. Though serum kisspeptin appears to be a potential biomarker to discriminate IUP from $\mathrm{SAB}$, it will be important to also follow patients with serial measurements for longitudinal assessment. Most patients in the IUP and SAB groups were African American, with only 4 Caucasian patients in the SAB group, and none in the IUP group, which limits our ability to detect an association if one exists. The nonpregnant controls were older than the IUP and SAB groups although kisspeptin did not appear to depend on maternal age. A larger study with a wider representation of patients across several racial and ethnic groups and similar maternal ages is needed to explore whether race, ethnicity, or maternal age impact kisspeptin trends. Asymptomatic pregnant controls were not included in the study, thus, the study is not generalizable to patients presenting in early pregnancy without symptoms. Future studies should also include asymptomatic pregnant patients to validate the serum kisspeptin assay in that population.

\section{Conclusions}

This study demonstrates that kisspeptin is detectable in serum in early pregnancy and can discriminate SAB from IUP. Future studies are needed to validate these findings, assess the applicability of the serum kisspeptin assay in other pregnancy outcomes including pregnancies after assisted reproductive techniques, ectopic pregnancy, and pregnancy of unknown location. Furthermore, it will be useful to compare the performance characteristics of the kisspeptin assay to other potential biomarkers of early pregnancy outcome. Ultimately, kisspeptin is a promising biomarker of pregnancy viability, and may have clinical utility in developing an accurate test of early pregnancy outcome in the future.

\section{Supplementary Material}

Refer to Web version on PubMed Central for supplementary material. 


\section{Acknowledgments}

Supported by 5R01HD076279 Biosignature of Ectopic Pregnancy - PI Kurt Barnhart and U10 HD027049 Cooperative Multicenter Reproductive Medicine Network.

The authors would like to thank Peter Takacs, MD, PhD of Eastern Virginia Medical School and Jared C. Robins, MD of the Northwestern University School of Medicine for their contributions.

We would also like to thank the Principal Investigators of the Reproductive Medicine Network and the Chair of the Steering Committee, Dr. Nannette Santoro, for their role in this project.

\section{References}

1. Ammon Avalos L, Galindo C, Li D-K. A systematic review to calculate background miscarriage rates using life table analysis. Birth Defects Research Part A: Clinical and Molecular Teratology. 2012; 94:417-23. [PubMed: 22511535]

2. Stephenson MD, Awartani KA, Robinson WP. Cytogenetic analysis of miscarriages from couples with recurrent miscarriage: a case-control study. Hum Reprod. 2002; 17:446-51. [PubMed: 11821293]

3. Horne AW, McBride R, Denison FC. Normally rising hCG does not predict live birth in women presenting with pain and bleeding in early pregnancy. European Journal of Obstetrics \& Gynecology and Reproductive Biology. 2011; 156:120-1. [PubMed: 21334129]

4. Doubilet PM, Benson CB, Bourne T, Blaivas M. Diagnostic Criteria for Nonviable Pregnancy Early in the First Trimester. New England Journal of Medicine. 2013; 369:1443-51. [PubMed: 24106937]

5. Valentin M-A, Ma S, Zhao A, Legay F, Avrameas A. Validation of immunoassay for protein biomarkers: Bioanalytical study plan implementation to support pre-clinical and clinical studies. Journal of Pharmaceutical and Biomedical Analysis. 2011; 55:869-77. [PubMed: 21530130]

6. Fisher AR, Sammel MD, Senapati S, Singer A, Barnhart KT. Temporal refinement does not affect predicted human chorionic gonadotropin rise in early pregnancy. Fertil Steril. 2016; 106:158-63. [PubMed: 27063601]

7. ACOG Committee on Practice Bulletins-Gynecology. ACOG Practice Bulletin no. 150. (Early pregnancy loss). Obstet Gynecol. 2015; 125:1258-67. [PubMed: 25932865]

8. Clarke H, Dhillo WS, Jayasena CN. Comprehensive Review on Kisspeptin and Its Role in Reproductive Disorders. Endocrinol Metab. 2015; 30:124-41.

9. Kavvasoglu S, Ozkan ZS, Kumbak B, Simsek M, Ilhan N. Association of kisspeptin-10 levels with abortus imminens: a preliminary study. Archives of Gynecology and Obstetrics. 2012; 285:649-53. [PubMed: 21847585]

10. Demirbilek H, Gonc EN, Ozon A, Alikasifoglu A, Kandemir N. Evaluation of serum kisspeptin levels in girls in the diagnosis of central precocious puberty and in the assessment of pubertal suppression. J Pediatr Endocrinol Metab. 2012; 25:313-6. [PubMed: 22768662]

11. Jayasena CN, Abbara A, Izzi-Engbeaya C, Comninos AN, Harvey RA, Maffe JG, et al. Reduced Levels of Plasma Kisspeptin During the Antenatal Booking Visit Are Associated With Increased Risk of Miscarriage. The Journal of Clinical Endocrinology \& Metabolism. 2014; 99:E2652-E60. [PubMed: 25127195]

12. Ohtaki T, Shintani Y, Honda S, Matsumoto H, Hori A, Kanehashi K, et al. Metastasis suppressor gene KiSS-1 encodes peptide ligand of a G-protein-coupled receptor. Nature. 2001; 411:613-7. [PubMed: 11385580]

13. Horikoshi Y, Matsumoto H, Takatsu Y, Ohtaki T, Kitada C, Usuki S, et al. Dramatic Elevation of Plasma Metastin Concentrations in Human Pregnancy: Metastin as a Novel Placenta-Derived Hormone in Humans. The Journal of Clinical Endocrinology \& Metabolism. 2003; 88:914-9. [PubMed: 12574233]

14. Park D-W, Lee S-K, Hong SR, Han A-R, Kwak-Kim J, Yang KM. Expression of Kisspeptin and its Receptor GPR54 in the First Trimester Trophoblast of Women with Recurrent Pregnancy Loss. American Journal of Reproductive Immunology. 2012; 67:132-9. [PubMed: 21996032] 
15. Bilban M, Ghaffari-Tabrizi N, Hintermann E, Bauer S, Molzer S, Zoratti C, et al. Kisspeptin-10, a KiSS-1/metastin-derived decapeptide, is a physiological invasion inhibitor of primary human trophoblasts. Journal of Cell Science. 2004; 117:1319-28. [PubMed: 15020672]

16. Francis VA, Abera AB, Matjila M, Millar RP, Katz AA. Kisspeptin regulation of genes involved in cell invasion and angiogenesis in first trimester human trophoblast cells. PLoS One. 2014; 9:e99680. [PubMed: 24923321]

17. Matjila M, Millar R, van der Spuy Z, Katz A. Elevated placental expression at the maternal-fetal interface but diminished maternal circulatory kisspeptin in preeclamptic pregnancies. Pregnancy Hypertension: An International Journal of Women's Cardiovascular Health. 2016; 6:79-87.

18. Muir AI, Chamberlain L, Elshourbagy NA, Michalovich D, Moore DJ, Calamari A, et al. AXOR12, a Novel Human G Protein-coupled Receptor, Activated by the Peptide KiSS-1. Journal of Biological Chemistry. 2001; 276:28969-75. [PubMed: 11387329]

19. Yu Z, Kastenmüller G, He Y, Belcredi P, Möller G, Prehn C, et al. Differences between Human Plasma and Serum Metabolite Profiles. PLOS ONE. 2011; 6:e21230. [PubMed: 21760889]

20. Ramachandran R, Patterson M, Murphy KG, Dhillo WS, Patel S, Kazarian A, et al. Preanalytical factors affecting RIA measurement of plasma kisspeptin. Clinical chemistry. 2008; 54:615-7. [PubMed: 18310154]

21. Bonassi S, Neri M, Puntoni R. Validation of biomarkers as early predictors of disease. Mutation Research/Fundamental and Molecular Mechanisms of Mutagenesis. 2001; 480-481:349-58. [PubMed: 11506827] 


\section{Capsule}

Serum kisspeptin levels are significantly higher in first trimester ongoing intrauterine pregnancies compared to miscarriages and the non-pregnant state. Kisspeptin may be a potential biomarker to predict pregnancy viability. 

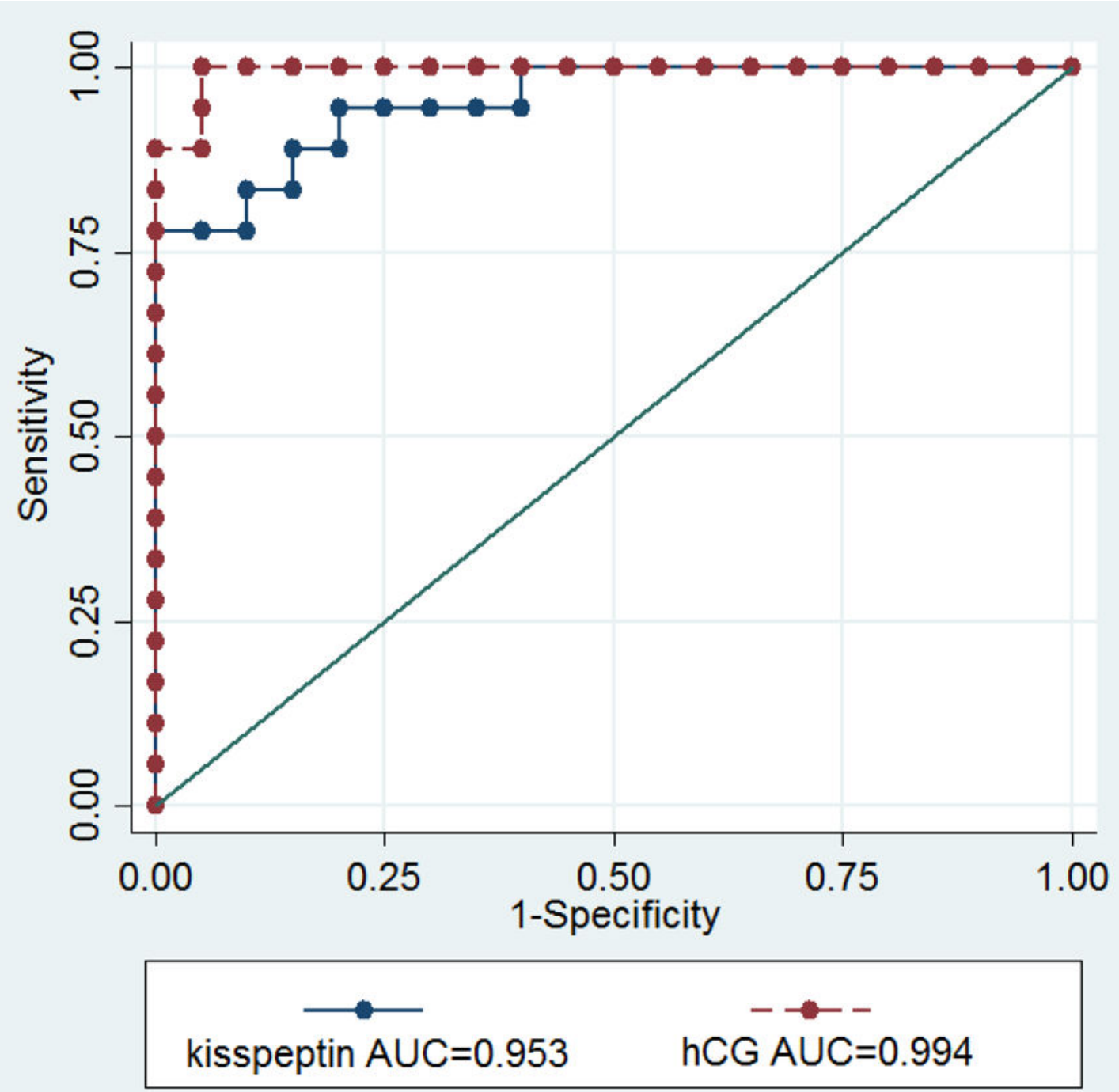

Figure 1.

Receiver Operator Curve analysis of the performance of serum kisspeptin and serum hCG. 


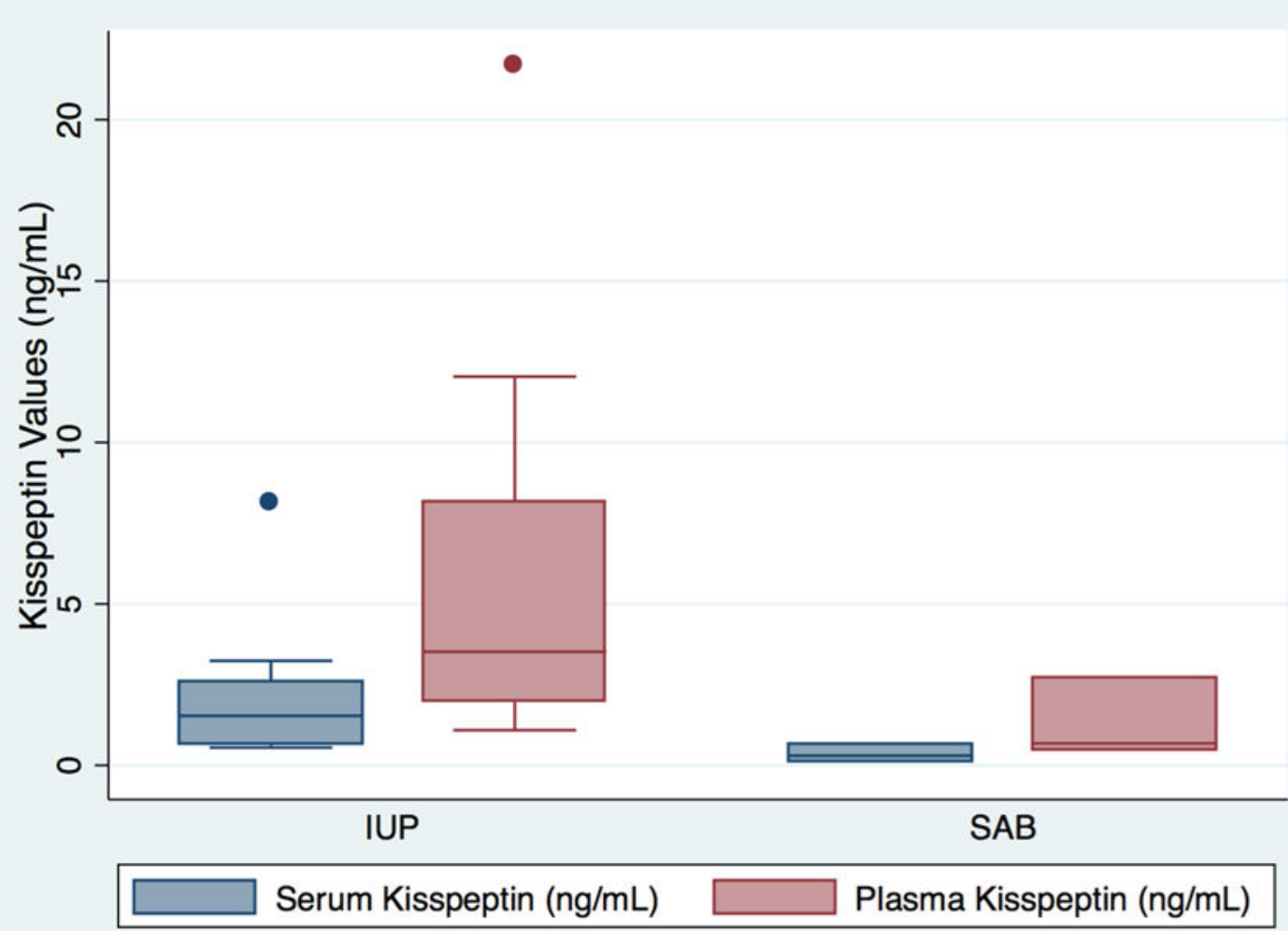

Figure 2.

Box Plot comparing serum and plasma kisspeptin values in patients with intrauterine pregnancies (IUP) and miscarriage (SAB). 


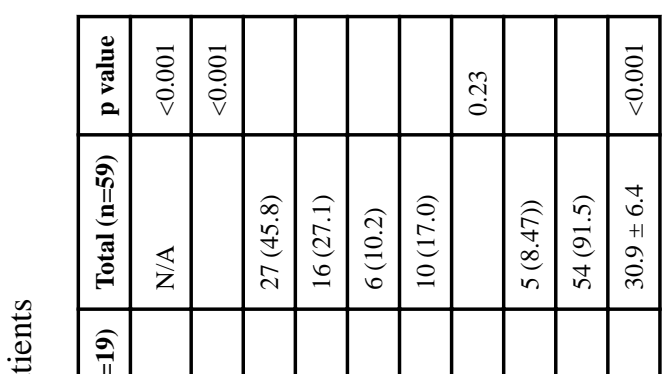




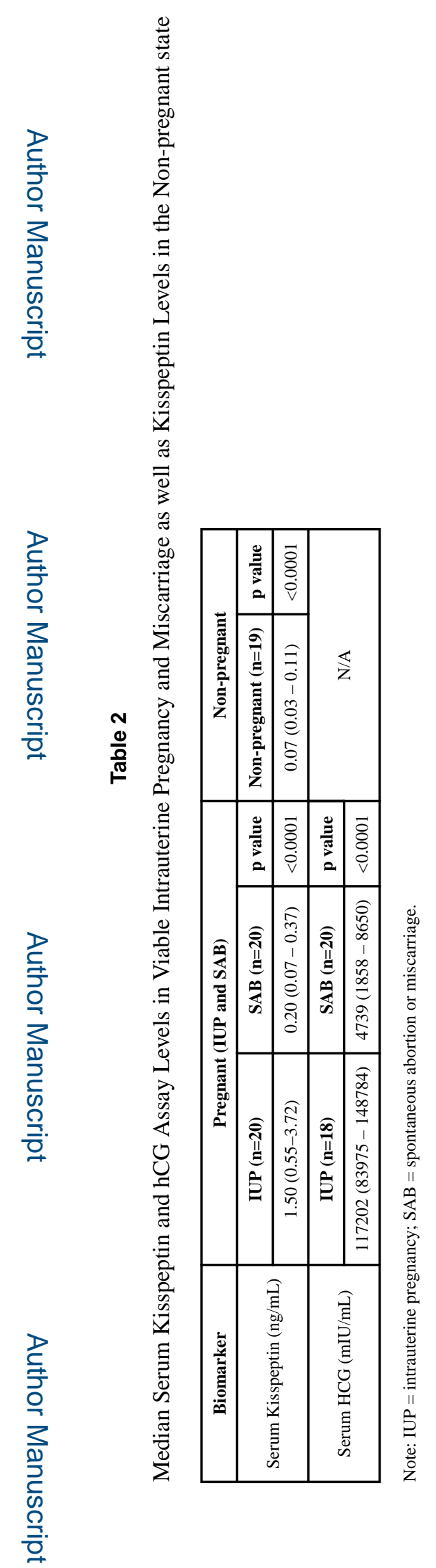

Fertil Steril. Author manuscript; available in PMC 2019 January 01. 